\title{
Transverse momentum resummation for $s$-channel single top quark production at the LHC
}

\author{
Peng Sun, ${ }^{1, *}$ Bin Yan, ${ }^{2, \dagger}$ and C.-P. Yuan ${ }^{2, \$}$ \\ ${ }^{1}$ Department of Physics and Institute of Theoretical Physics, Nanjing Normal University, \\ Nanjing, Jiangsu 210023, China \\ ${ }^{2}$ Department of Physics and Astronomy, Michigan State University, East Lansing, Michigan 48824, USA
}

(Received 13 November 2018; published 15 February 2019)

\begin{abstract}
We study the soft gluon radiation effects for the $s$-channel single top quark production at the LHC. By applying the transverse momentum dependent factorization formalism, the large logarithms about the small total transverse momentum $\left(q_{\perp}\right)$ of the single-top plus one-jet final state system are resummed to all orders in the expansion of the strong interaction coupling at the accuracy of next-to-leading Logarithm (NLL). We compare our numerical results with PYTHIA and find that both the $q_{\perp}$ and $\phi^{*}$ observables from PYTHIA are consistent with our prediction. Furthermore, we point out that the soft gluon radiation effects from the final state become significant in this process, especially for the boosted kinematical region.
\end{abstract}

DOI: 10.1103/PhysRevD.99.034008

\section{INTRODUCTION}

Single top quark production is an important source of top quarks at the Large Hadron Collider (LHC). There are three production modes, $s$-channel, $t$-channel and $t W$ associated production. In addition to measuring the $V_{t b}$ CabibboKobayashi-Maskawa (CKM) matrix element [1,2], they are also sensitive to different kinds of new physics (NP) models beyond the standard model (SM) [1-35], such as new heavy gauge boson $W^{\prime}$ [16-18], fermions [21-23], scalars [19,20], and $W t b$ anomalous couplings $[1,24-38]$. Compared with the $t$-channel and $t W$ associated production, the $s$-channel single top quark event is more sensitive to NP effects induced by heavy resonance states. Therefore, precisely study the single top quark production processes at the LHC become a vital task to test the SM and to search for new heavy particles. Recently, both the ATLAS and CMS Collaborations have conducted search for new particles through $s$-channel single top quark event at the $13 \mathrm{TeV}$ LHC, and concluded that their masses should be larger than about $\mathrm{TeV}$ scale $[39,40]$.

To further test the SM and search for NP through the single top quark processes, we should improve the accuracy of the theoretical prediction on its cross section and

\footnotetext{
pengsun@msu.edu yanbin1@msu.edu yuan@pa.msu.edu
}

Published by the American Physical Society under the terms of the Creative Commons Attribution 4.0 International license. Further distribution of this work must maintain attribution to the author(s) and the published article's title, journal citation, and DOI. Funded by SCOAP. kinematical distributions. The next-to-leading-order (NLO) QCD correction to the single top quark production has been widely discussed in the literatures [41-56]. The dominant part of the next-to-next-to-leading-order (NNLO) QCD corrections to predicting the detailed kinematical distributions, including proper spin correction, in $s$-channel and $t$-channel single top events, have also been discussed in Refs. [57-60]. To go beyond the fixed-order calculations, the threshold resummation technique is also widely discussed to improve the prediction on the single-top inclusive production rate [61-67]. The accuracy has reached to the next-to-leading-logarithm (NLL) and next-to-nextto-leading-logarithm (NNLL). Recently, the transverse momentum resummation formalism was proposed in Ref. [68] to improve the kinematical distributions of $t$-channel single top events. It shows that the sub-leading logarithms from the color correlation between the initial and final states play an important role when the final state jet is required to be in the forward region, where the resummation prediction is noticeably different from the PYTHIA parton shower results. Motivated by this, it is important to check on the kinematical distributions of $s$-channel single top quark events predicted by PYTHIA.

In this work, we apply the transverse momentum dependent (TMD) resummation technique to study the kinematical distribution of $s$-channel single top quark events,

$$
p+p \rightarrow W^{ \pm \star} \rightarrow t(\bar{t})+\mathrm{jet}+X .
$$

The large logarithms $\ln \left(Q^{2} / q_{\perp}^{2}\right)$, with $Q \gg q_{\perp}$ have been resummed to NLL accuracy, where $Q$ and $q_{\perp}$ are the invariant mass and the total transverse momentum of the top quark and jet system, respectively. The TMD 
resummation framework has been widely discussed in the color singlet processes [69-71]. For the processes with more complex color structures, like the heavy colored particle production was discussed in Refs. [72-74]. Recently, the TMD resummation formalism has been extended to discuss processes involving multijets in the final state; e.g., dijet production [75,76], Higgs plus one and two jets production [77-80], $Z$ boson and jet associated production [81] and $t$-channel single top quark production [68]. The soft gluon radiation from the final state will generate additional large logarithm $\ln \left(Q^{2} / q_{\perp}^{2}\right)$ when gluons are radiated outside the observed jet cone. Such logarithms can be resummed under the modified TMD resummation formalism. As to be shown below, the location and height of the Sudakov peak, in the $q_{\perp}$ distribution of $s$-channel single top quark events, strongly depends on the final state soft gluon radiation. Its effects could be enhanced largely when we focus on the boosted kinematical region of the final state. In contrast to the findings in the $t$-channel single-top production process, we find that the resummation calculation in the $s$-channel singletop process agrees well with PYTHIA prediction.

\section{TMD FACTORIZATION}

The differential cross section for $p p \rightarrow W^{ \pm \star} \rightarrow t(\bar{t})+$ jet $+X$ can be written as

$$
\begin{aligned}
& \frac{d^{4} \sigma}{d y_{t} d y_{J} d P_{J \perp}^{2} d^{2} q_{\perp}} \\
& =\sum_{a b}\left[\int \frac{d^{2} \vec{b}}{(2 \pi)^{2}} e^{-i \vec{q}_{\perp} \cdot \vec{b}} W_{a b \rightarrow t J}\left(x_{1}, x_{2}, \mathbf{b}\right)+Y_{a b \rightarrow t J}\right],
\end{aligned}
$$

where $y_{t}$ and $y_{J}$ denote rapidity for the top quark and the jet, respectively; $P_{J \perp}$ and $q_{\perp}$ are the transverse momenta of the jet and total transverse momentum of the top quark and the jet system, i.e., $\vec{q}_{\perp}=\vec{P}_{t \perp}+\vec{P}_{J \perp}$, respectively. The $W_{a b \rightarrow t J}$ term contains all order resummation and $Y_{a b \rightarrow t J}$ term accounts for the difference between the expansion of resummation part and the fixed-order corrections, and $x_{1}$, $x_{2}$ are momentum fractions of the incoming hadrons carried by the partons,

$$
x_{1,2}=\frac{\sqrt{m_{t}^{2}+P_{t \perp}^{2}} e^{ \pm y_{t}}+\sqrt{P_{J \perp}^{2}} e^{ \pm y_{J}}}{\sqrt{S}},
$$

where $m_{t}$ and $S$ are the top quark mass and the squared collider energy, respectively.

The all order resummation result for $W$-piece can be written as

$$
\begin{aligned}
W_{a b \rightarrow t J}\left(x_{1}, x_{2}, \mathbf{b}\right)= & x_{1} f_{a}\left(x_{1}, \mu_{F}=b_{0} / b_{*}\right) \\
& \times x_{2} f_{b}\left(x_{2}, \mu_{F}=b_{0} / b_{*}\right) \\
& \times e^{-S_{\text {Sud }}\left(Q^{2}, \mu_{\text {Res }}, b_{*}\right)} e^{-\mathcal{F}_{N P}\left(Q^{2}, \mathbf{b}\right)} \\
& \times H_{a b \rightarrow t J}\left(\mu_{\text {Res }}, \mu_{\text {Ren }}\right) S_{a b \rightarrow t J}\left(b_{0} / b_{*}\right),
\end{aligned}
$$

where $Q^{2}=\hat{s}=x_{1} x_{2} S, b_{0}=2 e^{-\gamma_{E}}$, with $\gamma_{E}$ being the Euler constant, $f_{a, b}\left(x, \mu_{F}\right)$ are parton distribution functions (PDFs) for the incoming partons $a$ and $b$, and $\mu_{\text {Res }}$ and $\mu_{\text {Ren }}$ represent the resummation and renormalization scales respectively in this process. Here, $b_{*}=\mathbf{b} / \sqrt{1+\mathbf{b}^{2} / b_{\max }^{2}}$ with $b_{\max }=1.5 \mathrm{GeV}^{-1}$, which is introduced to factor out the nonperturbative contribution $e^{-\mathcal{F}_{\mathrm{NP}}\left(Q^{2}, b\right)}$, arising from the large $\mathbf{b}$ region (with $\mathbf{b} \gg b_{*}$ ) [82-85],

$$
\mathcal{F}_{\mathrm{NP}}\left(Q^{2}, \mathbf{b}\right)=g_{1} \mathbf{b}^{2}+g_{2} \ln \frac{Q}{Q_{0}} \ln \frac{\mathbf{b}}{b_{*}},
$$

where $g_{1}=0.21, g_{2}=0.84$ and $Q_{0}^{2}=2.4 \mathrm{GeV}^{2}$ [85]. $H_{a b \rightarrow t J}$ and $S_{a b \rightarrow t J}$ are the hard and soft factors for this process. Similar to the $t$-channel single-top production process, there are two orthogonal color configurations in the $s$-channel single-top production process and the resummation calculation should be carried out in the color space with matrix form $[66,68]$. However, since the color-octet component in this process is much smaller than the color singlet component, we shall only include the color singlet component in our calculation as to be shown below. By applying the Catani-De Florian-Grazzini (CFG) scheme [86] and the TMD factorization in the Collins 2011 scheme [87], we obtain the hard factor $H_{a b \rightarrow t J}$, at the NLO level,

$$
\begin{aligned}
H_{a b \rightarrow t J}^{(1)}= & \frac{\alpha_{s}\left(\mu_{\text {Ren }}\right)}{2 \pi} C_{F} H^{(0)}\left[-\ln ^{2}(\lambda-1)-\frac{\ln (\lambda-1)}{\lambda}-2 \ln (\lambda-1)-2 \ln (\lambda-1) \ln \frac{\hat{s}}{m_{t}^{2}}+\ln \frac{\mu_{\text {Res }}^{2}}{\hat{s}}\left(-2 \ln (\lambda-1)-\ln \frac{\hat{s}}{m_{t}^{2}}-\frac{11}{2}\right)\right. \\
& \left.-\frac{1}{2} \ln ^{2} \frac{\hat{s}}{m_{t}^{2}}-\frac{5}{2} \ln \frac{\hat{s}}{m_{t}^{2}}-\frac{3}{2} \ln \frac{P_{J \perp}^{2} R^{2}}{\mu_{\text {Res }}^{2}}+2 \operatorname{Li}_{2}(\lambda)+\frac{1}{2} \ln ^{2} \frac{P_{J \perp}^{2} R^{2}}{\mu_{\text {Res }}^{2}}-\frac{3}{2} \ln ^{2} \frac{\mu_{\text {Res }}^{2}}{\hat{s}}+\frac{4 \pi^{2}}{3}-\frac{15}{2}\right]+\delta H^{(1),}
\end{aligned}
$$

where $\lambda=\hat{s} /\left(\hat{s}-m_{t}^{2}\right), R$ denotes the jet cone size of the final state jet. Both the loop correction and jet function have been included in the above hard factor. For the jet function calculation, the dimensional regularization and anti- $k_{T}$ jet algorithm are adopted in our calculation [76,88], and an off-shell mass is assigned to the light jet to regulate the light 
cone singularity in the soft factor calculation. The different treatment of the jet part in the jet function and the soft factor leads to a finite contribution in the hard factor, which does not depending on the jet size. Numerically, it is found to be approximately $\frac{\alpha_{s}}{2 \pi} C_{F} \frac{\pi^{2}}{6}$ for quark jet [78]. This additional factor has been considered as part of $H^{(1)}$. The leading order hard matrix element is,

$$
H^{(0)}(i j \rightarrow t \bar{b})=\frac{g^{4} \hat{t}\left(\hat{t}-m_{t}^{2}\right)}{4\left(\hat{s}-m_{W}^{2}\right)^{2}}\left|V_{i j}\right|^{2}\left|V_{t b}\right|^{2},
$$

where $g$ is $S U(2)_{L}$ gauge coupling. The CKM matrix element $V_{i j}$ needs to change for the corresponding incoming partons, and $m_{W}$ is $W$-boson mass. The $\delta H^{(1)}$ term is not proportional to the leading order matrix element,

$$
\delta H^{(1)}=\frac{\alpha_{s}}{2 \pi} \frac{1}{4} \frac{g^{4} C_{F} m_{t}^{2}}{\left(\hat{s}-m_{W}^{2}\right)^{2}} \frac{\hat{t} \hat{u}}{\hat{s}} \ln \frac{m_{t}^{2}}{\hat{s}-m_{t}^{2}}\left|V_{u d}\right|^{2}\left|V_{t b}\right|^{2},
$$

where $\hat{t}=\left(p_{u}-p_{\bar{b}}\right)^{2}$ and $\hat{u}=\left(p_{\bar{d}}-p_{\bar{b}}\right)^{2}$.

The Sudakov form factor $\mathcal{S}_{\text {Sud }}$ resums the leading double logarithm and the sub-leading logarithms,

$$
\begin{aligned}
S_{\text {Sud }}\left(Q^{2}, \mu_{\text {Res }}, b_{*}\right)= & \int_{b_{0}^{2} / b_{*}^{2}}^{\mu_{\text {Res }}^{2}} \frac{d \mu^{2}}{\mu^{2}}\left[\ln \left(\frac{Q^{2}}{\mu^{2}}\right) A+B_{1}+B_{2}\right. \\
& \left.+D_{1} \ln \frac{Q^{2}-m_{t}^{2}}{P_{J \perp}^{2} R^{2}}+D_{2} \ln \frac{Q^{2}-m_{t}^{2}}{m_{t}^{2}}\right],
\end{aligned}
$$

where the parameters $A, B_{1}, B_{2}, D_{1}$ and $D_{2}$ can be expanded perturbatively in $\alpha_{s}$. At one-loop order,

$$
\begin{aligned}
A=C_{F} \frac{\alpha_{s}}{\pi}, & B_{1}=-C_{F} \frac{3 \alpha_{s}}{2 \pi}, \\
B_{2}=-C_{F} \frac{\alpha_{s}}{2 \pi}, & D_{1}=D_{2}=C_{F} \frac{\alpha_{s}}{2 \pi},
\end{aligned}
$$

with $C_{F}=4 / 3$ in QCD interaction. In our numerical calculation, we will also include the $A^{(2)}$ contribution since it is associated with the incoming parton distributions and universal for all processes [86]. The coefficients $A$ and $B_{1}$
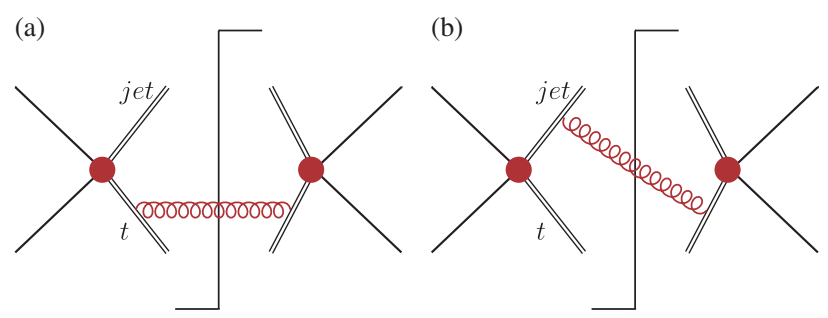

FIG. 1. Feynman diagrams contributing to the soft functions at the NLO.

come from the energy evolution effect in the TMD PDFs [89], so that they only depend on the flavor of the incoming partons of the leading order scattering processes. The coefficient $B_{2}$ describes the soft gluon emission from the final state top quark. The factor $D_{1}$ and $D_{2}$ quantifies the effect of soft gluon radiation between the top quark and jet in the final state.

The soft function $S_{a b \rightarrow t J}(\mu)$ at the scale $\mu$ can be calculated based on the method in Ref. [76]. At one loop order, it is

$$
\begin{aligned}
S_{a b \rightarrow t J}^{(1)}(\mu) & =-\frac{\alpha_{s}}{2 \pi} C_{F}\left[-1+\ln \frac{Q^{2}-m_{t}^{2}}{m_{t}^{2}}+\ln \frac{Q^{2}-m_{t}^{2}}{P_{J \perp^{2}} R^{2}}\right] \ln \left(\frac{b_{*}^{2}}{b_{0}^{2}} \mu^{2}\right) \\
& -\frac{\alpha_{s}}{2 \pi} C_{F}\left[\ln \frac{m_{t}^{2}}{m_{t}^{2}+P_{J \perp}^{2}}+I_{34}\right] .
\end{aligned}
$$

Hence, $S_{a b \rightarrow t J}^{(1)}\left(b_{0} / b_{*}\right)$ in Eq. (4), evaluated at $\mu=b_{0} / b_{*}$, is

$$
S_{a b \rightarrow t J}^{(1)}\left(b_{0} / b_{*}\right)=-\frac{\alpha_{s}}{2 \pi} C_{F}\left[\ln \frac{m_{t}^{2}}{m_{t}^{2}+P_{J \perp}^{2}}+I_{34}\right],
$$

where the first term originates from the contribution of the final state top quark line, as shown in Fig. 1(a), and $I_{34}$ represents the contribution of soft gluon radiation between the final state jet and top quark lines as shown in Fig. 1(b). In the narrow jet approximation; i.e., $P_{J \perp} R \rightarrow 0, I_{34}$ can be written as

$$
\begin{aligned}
I_{34}= & -\mathrm{Li}_{2} \frac{m_{t}^{2}+\hat{t}-\hat{u}}{\hat{t}}-\mathrm{Li}_{2} \frac{\left(2 m_{t}^{2}-\hat{s}\right)\left(m_{t}^{2}-\hat{t}\right)}{\hat{s} \hat{t}}+\mathrm{Li}_{2} \frac{\left(\hat{s}-2 m_{t}^{2}\right) \hat{t}}{\hat{s} \hat{u}}-\ln \frac{m_{t}^{2}-\hat{u}}{m_{t}^{2}+\hat{t}-\hat{u}} \ln \frac{-m_{t}^{2}\left(m_{t}^{2}+\hat{t}-\hat{u}\right)}{\hat{s} \hat{u}} \\
& +\ln \frac{-\hat{t}}{m_{t}^{2}+\hat{t}-\hat{u}} \ln \frac{\left(m_{t}^{2}-\hat{s}\right)\left(m_{t}^{2}+\hat{t}-\hat{u}\right)}{\hat{s} \hat{u}}+(\hat{t} \leftrightarrow \hat{u})-\ln \frac{\hat{s}-m_{t}^{2}}{m_{t}^{2}} \ln \frac{\hat{t} \hat{u}}{m_{t}^{4}-(\hat{t}-\hat{u})^{2}} \\
& -\ln \frac{P_{J \perp}^{2} R^{2} \hat{s}}{\hat{t} \hat{u}} \ln \frac{\hat{s}-m_{t}^{2}}{-P_{J \perp}^{2} R^{2}}-\frac{1}{2} \ln ^{2} \frac{P_{J \perp}^{2} R^{2}}{\hat{s}-2 m_{t}^{2}}-\frac{1}{2} \ln ^{2} \frac{m_{t}^{2}}{2 m_{t}^{2}-\hat{s}}+\frac{1}{2} \ln ^{2} \frac{\hat{s}-m_{t}^{2}}{2 m_{t}^{2}-\hat{s}}-\ln \frac{\hat{s}-m_{t}^{2}}{2 m_{t}^{2}-\hat{s}} \ln \frac{P_{J \perp}^{2} R^{2}}{\hat{s}-2 m_{t}^{2}} \\
& +2 \ln \frac{\hat{s}-m_{t}^{2}}{2 m_{t}^{2}-\hat{s}} \ln \frac{P_{J \perp}^{2} R^{2}}{\hat{s}-m_{t}^{2}}+\ln \frac{m_{t}^{2}}{2 m_{t}^{2}-\hat{s}} \ln \frac{m_{t}^{2} \hat{s}}{\hat{t} \hat{u}}-2 \ln \frac{2 m_{t}^{2}-\hat{s}}{m_{t}^{2}-\hat{s}} \ln \frac{m_{t}^{2}}{2 m_{t}^{2}-\hat{s}}-2 \operatorname{Li}_{2} \frac{m_{t}^{2}}{\hat{s}-m_{t}^{2}}-\frac{\pi^{2}}{3}+\mathcal{O}(\cdots),
\end{aligned}
$$


where the $(\cdots)$ term contains contributions proportional to $P_{J \perp} R$, and will be included in the following numerical calculation.

We should note that the nonglobal logarithms (NGLs) could also contribute to this process. The NGLs arise from some special kinematics of two soft gluon radiations, in which the first one is radiated outside of the jet which subsequently radiates a second gluon into the jet [90-93]. Numerically, the NGLs are negligible in this process since it starts at $\mathcal{O}\left(\alpha_{s}^{2}\right)$ [94]. Therefore we will ignore their contributions in the following phenomenology discussion.

\section{PHENOMENOLOGY OF SINGLE TOP QUARK PRODUCTION}

Before presenting the result of resummation effects on the kinematical distributions of the $s$-channel single top quark events, it is important to cross-check the total cross section with the fixed-order calculation. In the resummation framework, the NLO total cross section can be divided into two parts, the small $q_{\perp}$ region, which can be obtained by integrating the distribution of the asymptotic part and virtual diagram contribution, and the large $q_{\perp}$ part, which is infrared safe and can be numerically calculated directly [95]. Thus, the NLO total cross section is given by

$\sigma_{\mathrm{NLO}}=\int_{0}^{q_{\perp, 0}^{2}} d q_{\perp}^{2} \frac{d \sigma_{\mathrm{NLO}}^{\text {virtual }+ \text { real }}}{d q_{\perp}^{2}}+\int_{q_{\perp, 0}^{2}}^{\infty} d q_{\perp}^{2} \frac{d \sigma_{\mathrm{NLO}}^{\text {real }}}{d q_{\perp}^{2}}$,

where $q_{\perp, 0}=1 \mathrm{GeV}$ labels the cutoff of $q_{\perp}$. In the above equation, the integrand of the first term was obtained by expanding the contribution from the $W$-term, cf. Eq. (4), up to order $\alpha_{s}$, but without including the $Y$-term contribution which is small for $q_{\perp}<1 \mathrm{GeV}$. The numerical result of Eq. (14) is found to be slightly different from the prediction of MCFM with $\mu_{\text {Ren }}=\mu_{F}=m_{t}$ [96], ranging from $1.8 \%$ for $R=0.4$ to $0.3 \%$ for $R=0.2$. Clearly, this discrepancy arises from the narrow jet approximation we made in our calculation. Following the procedure of Ref. [78], we parameterize this difference as function of $R$ : $H^{(0)} \frac{\alpha_{s}}{2 \pi}\left(-1.3 R+12.0 R^{2}\right)$ for the range of $0.2<R<0.6$, which has been included in $H^{(1)}$.

Figure 2 shows various differential cross sections of the $s$-channel single top quark (a) and anti-top quark (b) production at the $\sqrt{S}=13 \mathrm{TeV}$ LHC with CT14NNLO PDFs [97], which were determined in the variable flavor general mass scheme (VFGM) up to five flavors. Here, we have included the contribution in which bottom quark is one of the incoming partons and taken to be massless in the constituent cross section calculations. The blue dashed line for asymptotic piece, red dotted line for NLO calculation, black solid line for our resummation prediction, and orange dot-dashed line for the $Y$-term. The asymptotic piece is the fixed-order expansion of Eq. (2) up to the $\alpha_{s}$ order. In our resummation calculation, the resummation scale $\left(\mu_{\text {Res }}\right)$ and
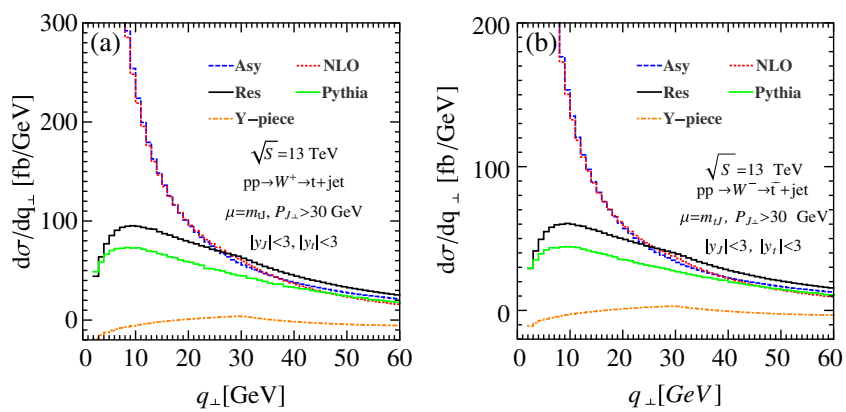

FIG. 2. The $q_{\perp}$ distribution from the asymptotic result (blue dashed line), NLO calculation (red dotted line), resummation prediction (black solid line), parton shower Monte Carlo prediction by PYTHIA8 (green solid line), and $Y$-term (orange dotdashed line) for the $s$-channel single top quark (a) and anti-top quark (b) production at the $\sqrt{S}=13 \mathrm{TeV}$ LHC with $\left|y_{J}\right|<3$, $\left|y_{t}\right|<3$ and $P_{J \perp}>30 \mathrm{GeV}$. The resummation and renormalization scales are choose as $\mu=\mu_{\text {Res }}=\mu_{\text {ren }}=m_{t J}=\left(p_{t}+p_{J}\right)^{2}$.

renormalization $\left(\mu_{\text {ren }}\right)$ scales are taken to be the invariant mass of top quark and jet $\left(m_{t J}=\left(p_{t}+p_{J}\right)^{2}\right)$. Similarly, the renormalization and factorization scales are also fixed to $m_{t J}$ in the fixed-order calculation. The cone size $R=0.4$ and anti- $k_{T}$ jet algorithm are used to define the observed jet. The following kinematic cuts are also required in our numerical calculation, $\left|y_{J}\right|<3,\left|y_{t}\right|<3$ and $P_{J \perp}>30 \mathrm{GeV}$. In the same figure, we also compared to the prediction from the parton shower event generator PYTHIA8 [98] (green solid line), which was calculated at the leading order, with CT14LO PDF. The uncertainties of the resummation predictions are estimated by varying the scale $\mu_{\text {Res }}=\mu_{\text {ren }}$ by a factor of two around the central value $m_{t J}$, which is shown in Fig. 3. Note that the uncertainty bands could be slight different if we vary the resummation and renormalization scales independently in the calculation. In Fig. 4, we compare the prediction from our resummation calculation to PYTHIA by taking the ratio of their $q_{\perp}$ differential distributions in Fig. 2. As shown, its ratio is not sensitive to $q_{\perp}$ for either single top (a) or anti-top quark (b) production when $q_{\perp}>10 \mathrm{GeV}$, but not for the small $q_{\perp}$ region.
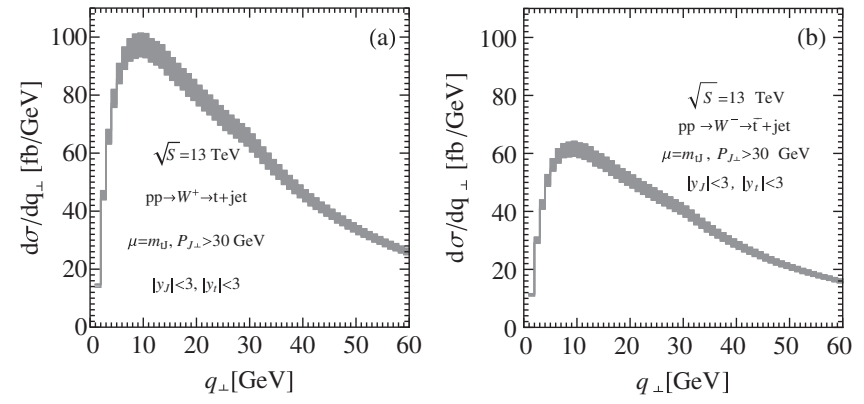

FIG. 3. The scale uncertainties for the $s$-channel single top quark (a) and anti-top quark (b) production at the $\sqrt{S}=13 \mathrm{TeV}$ LHC with $\left|y_{J}\right|<3,\left|y_{t}\right|<3$ and $P_{J \perp}>30 \mathrm{GeV}$. The resummation and renormalization scales are varied from $m_{t J} / 2$ to $2 m_{t J}$. 

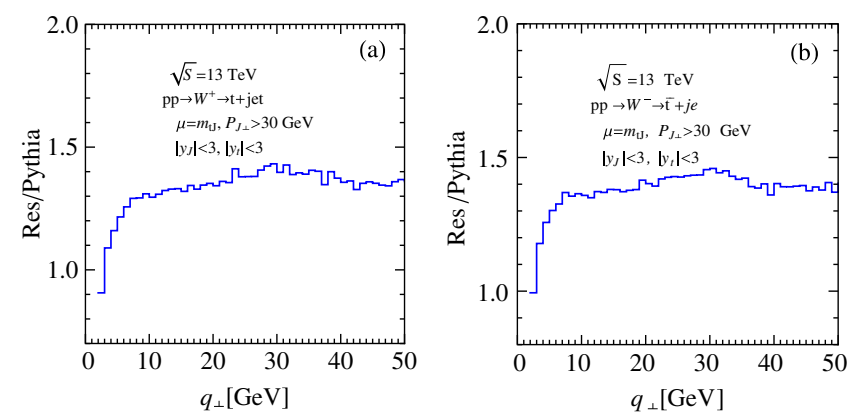

FIG. 4. The ratio of the resummation and PYTHIA8 prediction for the $s$-channel single top quark (a) and anti-top quark (b) production at the $\sqrt{S}=13 \mathrm{TeV}$ LHC with $\left|y_{J}\right|<3,\left|y_{t}\right|<3$ and $P_{J \perp}>30 \mathrm{GeV}$. The resummation and renormalization scales are choose as $\mu=\mu_{\mathrm{Res}}=\mu_{\mathrm{ren}}=m_{t J}$.

Hence, they predict almost the same shape in $q_{\perp}$ distribution, while they predict different fiducial total cross section because PYTHIA prediction includes only leading order matrix element and is calculated with CT14LO PDF. It would also be interesting to compare our resummation prediction with that from a parton shower Monte Carlo event generator with NLO matrix element which is however beyond the scope of this work.

In order to estimate the soft gluon radiation effects from the final state, we show various normalized $W$-pieces predictions in Fig. 5. The red dashed line denotes the $W$-piece prediction when we only keep the Drell-Yan-like Sudakov factor; i.e., the parameters in Eq. (10) are changed as $D_{1}=D_{2}=0$ and $B_{1}=-\frac{3}{2} C_{F} \frac{\alpha_{s}}{\pi}$ (labeled as " $W$-term DY" in Fig. 5). The dotted black (labeled as " $W$-term cut") and solid blue lines label the results from the $W$-piece including all the Sudakov factor with and without $m_{t J}>$ $1 \mathrm{TeV}$ cut, respectively. It shows that the soft gluon radiation effects from final state are significant in this
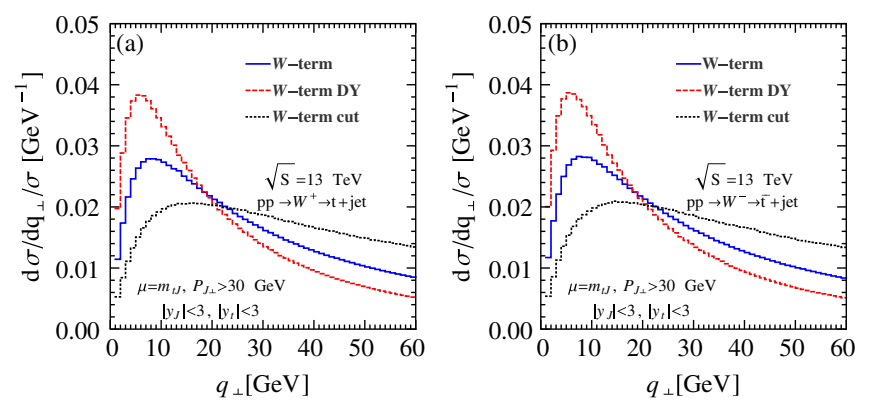

FIG. 5. The normalized $W$-piece prediction for the $s$-channel single top quark (a) and anti-top quark (b) production at the $\sqrt{S}=$ $13 \mathrm{TeV}$ LHC with $\left|y_{J}\right|<3,\left|y_{t}\right|<3$ and $P_{J \perp}>30 \mathrm{GeV}$. The red-dashed line denotes the $W$-piece prediction with only DrellYan-like Sudakov factor, while the dotted black and solid blue lines label the results from the $W$-piece with and without $m_{t J}>1 \mathrm{TeV}$ cut. The resummation and renormalization scales are choose as $\mu=\mu_{\text {Res }}=\mu_{\text {ren }}=m_{t J}$. case, especially when we focus on the boosted kinematical phase space region where the term $\left(\ln \frac{Q^{2}-m_{t}^{2}}{P_{J \perp}^{2} R^{2}}+\ln \frac{Q^{2}-m_{t}^{2}}{m_{t}^{2}}\right)$ in the Sudakov factor becomes large. Consequently, the $q_{\perp}$ distribution peaks at a larger value.

Similar to the $t$-channel single top quark production, we can define the $\phi^{*}$ observable to study the soft gluon radiation effects $[68,99]$. Since the $\phi^{*}$ only depends on the moving directions (not energies) of the final state jet and top quark, it might reduce the experimental uncertainties and provide a better measurement for probing the soft gluon radiation effects. The definition is,

$$
\phi^{*}=\tan \left(\frac{\pi-\Delta \phi}{2}\right) \sin \theta_{\eta}^{*},
$$

where $\Delta \phi$ is the azimuthal angle separation in radians between the jet and top quark. The angle $\theta_{\eta}^{*}$ is defined as

$$
\cos \theta_{\eta}^{*}=\tanh \left[\frac{\eta_{J}-\eta_{t}}{2}\right],
$$

where $\eta_{J}$ and $\eta_{t}$ are the pseudorapidities of the jet and top quark, respectively. As show in Fig. 6, the prediction of PYTHIA (black dotted line) and our resummation calculation (blue solid line; labeled as "Res") are consistent with each other. However, if we only keep the Drell-Yan-like Sudakov factor in the $W$-term, the $\phi^{*}$ tends to a smaller value (red dashed line; labeled as "Res-DY" in Fig. 6). It could be understood from the $W$-piece prediction in Fig. 5, where the Sudakov factor from the final state soft gluon radiation would push the $q_{\perp}$ distribution to peak at a larger $q_{\perp}$ value. Because large $q_{\perp}$ value corresponds to the large $\phi^{*}$ value, the final state soft gluon radiation would push the average $\phi^{*}$ value to a larger value as compared to the Drell-Yan-like Sudakov factor case.
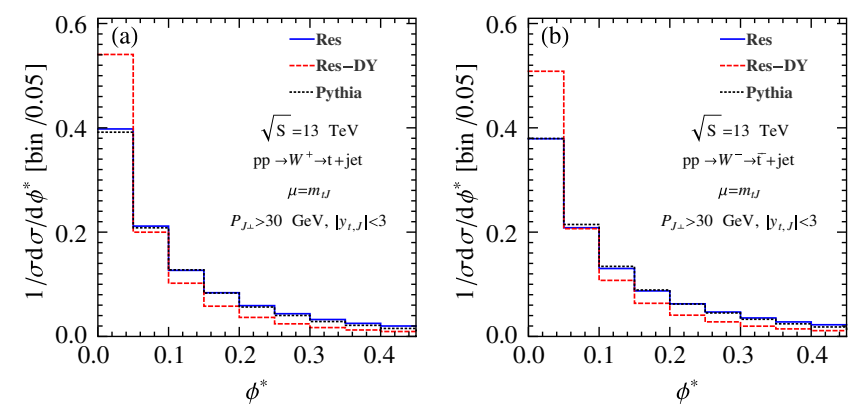

FIG. 6. The normalized distribution of $\phi^{*}$ for top quark (a) and anti-top quark (b) production at the $13 \mathrm{TeV}$ LHC with $\left|y_{t, J}\right|<3$ and $P_{J \perp}>30 \mathrm{GeV}$. The resummation and renormalization scales are choose as $\mu=\mu_{\text {Res }}=\mu_{\text {ren }}=m_{t J}$. The blue solid and red dashed line represent the resummation prediction with full Sudakov factor and only Drell-Yan-like Sudakov factor, respectively. The black dotted line labels the prediction from PYTHIA8. 


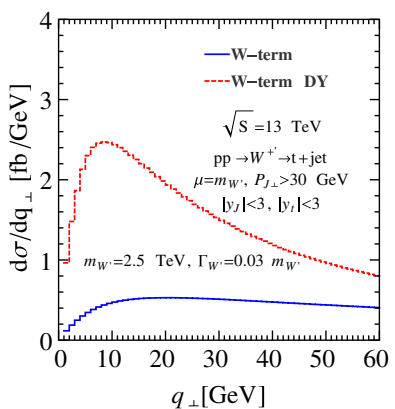

FIG. 7. The prediction for $p p \rightarrow W^{\prime+} \rightarrow t+$ jet at the $\sqrt{S}=$ $13 \mathrm{TeV}$ LHC with $\left|y_{J}\right|<3,\left|y_{t}\right|<3$ and $P_{J \perp}>30 \mathrm{GeV}$. The red-dashed line denotes the $W$-piece prediction with only DrellYan-like Sudakov factor, while the solid blue line labels the result from the $W$-piece with full Sudakov factor. The resummation and renormalization scales are choose as $\mu=\mu_{\text {Res }}=\mu_{\text {ren }}=m_{W^{\prime}}$.

\section{PHENOMENOLOGY OF TOP-FLAVOR $W^{\prime}$ PRODUCTION}

In many new physics models, extra heavy particles would favor decay into top quark and jet, e.g., the $W^{\prime}$ in top-flavor models $[16-18,100,101]$ or a charged Higgs $H^{+}$ in the general two Higgs doublet models [14]. When $m_{W^{\prime} / H^{+}} \gg m_{t}$, the decayed top quark and jet are highly boosted. As we discussed in the last section, the Sudakov enhancement from the final state soft gluon radiation would become more important when top quark and jet are highly boosted. In this section, we use the sequential standard model (SSM) $W^{\prime}$ as an example to discuss the final state soft gluon radiation effects on the kinematical distribution of the $W^{\prime}$. Our results can easily be extended to the general $W^{\prime}$ new physics models. The effective Lagrangian related to our study is

$$
\mathcal{L}=\frac{g}{\sqrt{2}} V_{i j} \bar{q}_{i} \gamma_{\mu} \frac{1-\gamma_{5}}{2} q_{j} W^{\prime \mu}
$$

The null result in the search of $W^{\prime}$ via the single top quark channel at the $13 \mathrm{TeV}$ LHC by ATLAS and CMS Collaborations impose a strong bound on $m_{W^{\prime}}$, which should be larger than $2-3 \mathrm{TeV}[39,40]$. In this work, we assume $m_{W^{\prime}}=2.5 \mathrm{TeV}$ and $\Gamma_{W^{\prime}}=0.03 m_{W^{\prime}}=75 \mathrm{GeV}$ as our benchmark point to study the soft gluon radiation effects on the transverse momentum distribution of $W^{\prime}$.

The $W$-term predictions with full Sudakov factor (blue solid line) and Drell-Yan-like Sudakov factor (red-dashed line) at the $13 \mathrm{TeV}$ LHC are shown in Fig. 7. Clearly, the soft gluon radiation effects from the final state jet and top quark are important for the search of heavy resonance states in $s$-channel single-top processes.

\section{CONCLUSION}

In this work, we studied the $q_{\perp}$ resummation effects for the $s$-channel single top quark production at the LHC based on the TMD factorization theorem. The large logarithm $\ln \left(Q^{2} / q_{\perp}^{2}\right)$ was resummed by renormalization group evolution at NLL accuracy. We also calculated the NLO total cross section derived from the resummation framework, while yields a slightly different result from the MCFM prediction due to the usage of narrow jet approximation in our resummation calculation. To ensure the correct NLO total cross section, we have added an additional term proportional to $H^{(0)}$ to account for the above difference in our resummation calculation. A detailed comparison between our theory calculation and PYTHIA8 prediction was also discussed. We find that both the total transverse momentum $\left(q_{\perp}\right)$ and $\phi^{*}$ distributions predicted by our theory calculation agree well with the PYTHIA8 prediction. Furthermore, the soft gluon radiation effects from the final state would change the shape of $q_{\perp}$ or $\phi^{*}$ distribution significantly, especially when the top quark and jet are highly boosted. Finally, we discussed the soft gluon radiation effects for the production of $W^{\prime}$ or $\mathrm{H}^{+}$boson which subsequently decays into a pair of top quark and jet.

\section{ACKNOWLEDGMENTS}

This work was supported by the U.S. National Science Foundation under Grant No. PHY-1719914. C.-P. Yuan is also grateful for the support from the Wu-Ki Tung endowed chair in particle physics.
[1] E. L. Berger, Q.-H. Cao, and I. Low, Phys. Rev. D 80, 074020 (2009).

[2] Q.-H. Cao and B. Yan, Phys. Rev. D 92, 094018 (2015).

[3] S. Dawson, Nucl. Phys. B249, 42 (1985).

[4] S. S. D. Willenbrock and D. A. Dicus, Phys. Rev. D 34, 155 (1986).

[5] S. Dawson and S. S. D. Willenbrock, Nucl. Phys. B284, 449 (1987).
[6] C. P. Yuan, Phys. Rev. D 41, 42 (1990).

[7] G. A. Ladinsky and C. P. Yuan, Phys. Rev. D 49, 4415 (1994).

[8] D. O. Carlson and C.P. Yuan, Phys. Lett. B 306, 386 (1993).

[9] D. O. Carlson and C.P. Yuan, in Top Quark Workshop Ames, Iowa, 1995 (1995), pp. 172-177.

[10] T. M. P. Tait and C. P. Yuan, Phys. Rev. D 55, 7300 (1997). 
[11] C. S. Li, R. J. Oakes, and J. M. Yang, Phys. Rev. D 55, 5780 (1997).

[12] C.-S. Li, R. J. Oakes, J.-M. Yang, and H.-Y. Zhou, Phys. Rev. D 57, 2009 (1998).

[13] T. M. P. Tait and C. P. Yuan, arXiv:hep-ph/9710372.

[14] H.-J. He and C. P. Yuan, Phys. Rev. Lett. 83, 28 (1999).

[15] T. M. P. Tait and C. P. Yuan, Phys. Rev. D 63, 014018 (2000).

[16] E. Malkawi, T. M. P. Tait, and C. P. Yuan, Phys. Lett. B 385, 304 (1996).

[17] K. Hsieh, K. Schmitz, J.-H. Yu, and C. P. Yuan, Phys. Rev. D 82, 035011 (2010).

[18] Q.-H. Cao, Z. Li, J.-H. Yu, and C. P. Yuan, Phys. Rev. D 86, 095010 (2012).

[19] E. Drueke, J. Nutter, R. Schwienhorst, N. Vignaroli, D. G. E. Walker, and J.-H. Yu, Phys. Rev. D 91, 054020 (2015).

[20] Q.-H. Cao, X. Wan, X.-p. Wang, and S.-h. Zhu, Phys. Rev. D 87, 055022 (2013).

[21] Q.-H. Cao, C. S. Li, and C. P. Yuan, Phys. Lett. B 668, 24 (2008).

[22] E. L. Berger, Q.-H. Cao, C.-R. Chen, and H. Zhang, Phys. Rev. D 83, 114026 (2011).

[23] E. L. Berger, Q.-H. Cao, J.-H. Yu, and C. P. Yuan, Phys. Rev. D 84, 095026 (2011).

[24] G. L. Kane, G. A. Ladinsky, and C. P. Yuan, Phys. Rev. D 45, 124 (1992).

[25] D. O. Carlson, E. Malkawi, and C. P. Yuan, Phys. Lett. B 337, 145 (1994).

[26] C.-R. Chen, F. Larios, and C. P. Yuan, Phys. Lett. B 631, 126 (2005).

[27] Q.-H. Cao, J. Wudka, and C. P. Yuan, Phys. Lett. B 658, 50 (2007).

[28] M. Fabbrichesi, M. Pinamonti, and A. Tonero, Eur. Phys. J. C 74, 3193 (2014).

[29] C. Bernardo, N. F. Castro, M. C. N. Fiolhais, H. Gonalves, A. G. C. Guerra, M. Oliveira, and A. Onofre, Phys. Rev. D 90, 113007 (2014).

[30] Q.-H. Cao, B. Yan, J.-H. Yu, and C. Zhang, Chin. Phys. C 41, 063101 (2017).

[31] A. Prasath V, R. M. Godbole, and S. D. Rindani, Eur. Phys. J. C 75, 402 (2015).

[32] Z. Hioki and K. Ohkuma, Phys. Lett. B 752, 128 (2016).

[33] C. Zhang, Phys. Rev. Lett. 116, 162002 (2016).

[34] J. L. Birman, F. Dliot, M. C. N. Fiolhais, A. Onofre, and C. M. Pease, Phys. Rev. D 93, 113021 (2016).

[35] E. Boos, V. Bunichev, L. Dudko, and M. Perfilov, Int. J. Mod. Phys. A 32, 1750008 (2017).

[36] J. A. Aguilar-Saavedra, Nucl. Phys. B804, 160 (2008).

[37] S. D. Rindani and P. Sharma, J. High Energy Phys. 11 (2011) 082.

[38] A. Jueid, Phys. Rev. D 98, 053006 (2018).

[39] A. M. Sirunyan et al. (CMS Collaboration), Phys. Lett. B 777, 39 (2018).

[40] M. Aaboud et al. (ATLAS Collaboration), Phys. Lett. B 781, 327 (2018).

[41] S. Zhu, Phys. Lett. B 524, 283 (2002); 537, 351(E) (2002).

[42] B. W. Harris, E. Laenen, L. Phaf, Z. Sullivan, and S. Weinzierl, Phys. Rev. D 66, 054024 (2002).

[43] Q.-H. Cao and C. P. Yuan, Phys. Rev. D 71, 054022 (2005).
[44] Q.-H. Cao, R. Schwienhorst, and C. P. Yuan, Phys. Rev. D 71, 054023 (2005).

[45] J. M. Campbell, R. K. Ellis, and F. Tramontano, Phys. Rev. D 70, 094012 (2004).

[46] Q.-H. Cao, R. Schwienhorst, J. A. Benitez, R. Brock, and C. P. Yuan, Phys. Rev. D 72, 094027 (2005).

[47] J. M. Campbell and F. Tramontano, Nucl. Phys. B726, 109 (2005).

[48] Q.-H. Cao, arXiv:0801.1539.

[49] S. Heim, Q.-H. Cao, R. Schwienhorst, and C. P. Yuan, Phys. Rev. D 81, 034005 (2010).

[50] J. M. Campbell, R. Frederix, F. Maltoni, and F. Tramontano, Phys. Rev. Lett. 102, 182003 (2009).

[51] R. Schwienhorst, C. P. Yuan, C. Mueller, and Q.-H. Cao, Phys. Rev. D 83, 034019 (2011).

[52] P. Falgari, P. Mellor, and A. Signer, Phys. Rev. D 82, 054028 (2010).

[53] S. Frixione, E. Laenen, P. Motylinski, and B. R. Webber, J. High Energy Phys. 03 (2006) 092.

[54] S. Alioli, P. Nason, C. Oleari, and E. Re, J. High Energy Phys. 09 (2009) 111; 02 (2010) 11.

[55] R. Frederix, E. Re, and P. Torrielli, J. High Energy Phys. 09 (2012) 130.

[56] R. Frederix, S. Frixione, A. S. Papanastasiou, S. Prestel, and P. Torrielli, J. High Energy Phys. 06 (2016) 027.

[57] M. Brucherseifer, F. Caola, and K. Melnikov, Phys. Lett. B 736, 58 (2014).

[58] E. L. Berger, J. Gao, C. P. Yuan, and H. X. Zhu, Phys. Rev. D 94, 071501 (2016).

[59] E. L. Berger, J. Gao, and H. X. Zhu, J. High Energy Phys. 11 (2017) 158.

[60] Z. L. Liu and J. Gao, Phys. Rev. D 98, 071501 (2018).

[61] N. Kidonakis, Phys. Rev. D 74, 114012 (2006).

[62] N. Kidonakis, Phys. Rev. D 75, 071501 (2007).

[63] N. Kidonakis, Phys. Rev. D 83, 091503 (2011).

[64] N. Kidonakis, Phys. Rev. D 88, 031504 (2013).

[65] N. Kidonakis, Phys. Rev. D 93, 054022 (2016).

[66] H. X. Zhu, C.S. Li, J. Wang, and J. J. Zhang, J. High Energy Phys. 02 (2011) 099.

[67] J. Wang, C. S. Li, H. X. Zhu, and J. J. Zhang, arXiv:1010 .4509 .

[68] Q.-H. Cao, P. Sun, B. Yan, C. P. Yuan, and F. Yuan, Phys. Rev. D 98, 054032 (2018).

[69] J. C. Collins and D. E. Soper, Nucl. Phys. B193, 381 (1981); B213, 545(E) (1983).

[70] J. C. Collins and D. E. Soper, Nucl. Phys. B197, 446(E) (1982).

[71] J. C. Collins, D. E. Soper, and G. F. Sterman, Nucl. Phys. B250, 199 (1985).

[72] H. X. Zhu, C. S. Li, H. T. Li, D. Y. Shao, and L. L. Yang, Phys. Rev. Lett. 110, 082001 (2013).

[73] H. T. Li, C. S. Li, D. Y. Shao, L. L. Yang, and H. X. Zhu, Phys. Rev. D 88, 074004 (2013).

[74] R. Zhu, P. Sun, and F. Yuan, Phys. Lett. B 727, 474 (2013).

[75] P. Sun, C. P. Yuan, and F. Yuan, Phys. Rev. Lett. 113, 232001 (2014).

[76] P. Sun, C. P. Yuan, and F. Yuan, Phys. Rev. D 92, 094007 (2015).

[77] P. Sun, C. P. Yuan, and F. Yuan, Phys. Rev. Lett. 114, 202001 (2015). 
[78] P. Sun, J. Isaacson, C. P. Yuan, and F. Yuan, Phys. Lett. B 769, 57 (2017).

[79] P. Sun, C. P. Yuan, and F. Yuan, Phys. Lett. B 762, 47 (2016).

[80] P. Sun, C. P. Yuan, and F. Yuan, arXiv:1802.02980.

[81] P. Sun, B. Yan, C. P. Yuan, and F. Yuan, arXiv:1810.03804.

[82] F. Landry, R. Brock, G. Ladinsky, and C. P. Yuan, Phys. Rev. D 63, 013004 (2000).

[83] F. Landry, R. Brock, P. M. Nadolsky, and C. P. Yuan, Phys. Rev. D 67, 073016 (2003).

[84] P. Sun, C. P. Yuan, and F. Yuan, Phys. Rev. D 88, 054008 (2013).

[85] P. Sun, J. Isaacson, C. P. Yuan, and F. Yuan, Int. J. Mod. Phys. A 33, 1841006 (2018).

[86] S. Catani, D. de Florian, and M. Grazzini, Nucl. Phys. B596, 299 (2001).

[87] J. Collins, Foundations of Perturbative QCD (Cambridge University Press, Cambridge, England, 2013).

[88] A. Mukherjee and W. Vogelsang, Phys. Rev. D 86, 094009 (2012).

[89] X.-d. Ji, J.-p. Ma, and F. Yuan, Phys. Rev. D 71, 034005 (2005).
[90] M. Dasgupta and G. P. Salam, Phys. Lett. B 512, 323 (2001).

[91] M. Dasgupta and G. P. Salam, J. High Energy Phys. 03 (2002) 017.

[92] A. Banfi and M. Dasgupta, J. High Energy Phys. 01 (2004) 027.

[93] J. R. Forshaw, A. Kyrieleis, and M. H. Seymour, J. High Energy Phys. 08 (2006) 059.

[94] P. Sun, C. P. Yuan, and F. Yuan (to be published).

[95] C. Balazs and C. P. Yuan, Phys. Rev. D 56, 5558 (1997).

[96] J. M. Campbell, R. K. Ellis, and W. T. Giele, Eur. Phys. J. C 75, 246 (2015).

[97] S. Dulat, T.-J. Hou, J. Gao, M. Guzzi, J. Huston, P. Nadolsky, J. Pumplin, C. Schmidt, D. Stump, and C. P. Yuan, Phys. Rev. D 93, 033006 (2016).

[98] T. Sjostrand, S. Mrenna, and P. Z. Skands, Comput. Phys. Commun. 178, 852 (2008).

[99] A. Banfi, S. Redford, M. Vesterinen, P. Waller, and T. R. Wyatt, Eur. Phys. J. C 71, 1600 (2011).

[100] X. Li and E. Ma, Phys. Rev. Lett. 47, 1788 (1981).

[101] H.-J. He, T. M. P. Tait, and C. P. Yuan, Phys. Rev. D 62, 011702 (2000). 